\title{
Programa Bolsa Família e estado nutricional infantil: desafios estratégicos
}

\author{
Bolsa Família Program and child nutritional status: \\ strategic challenges
}

Fabiana de Cássia Carvalho Oliveira ${ }^{1}$

Rosângela Minardi Mitre Cotta ${ }^{1}$

Luciana Ferreira da Rocha Sant'Ana ${ }^{1}$

Silvia Eloíza Priore ${ }^{1}$

Sylvia do Carmo Castro Franceschini ${ }^{1}$

${ }^{1}$ Departamento de Nutrição e Saúde (DNS),

Universidade Federal de

Viçosa. Avenida PH Rolfs $\mathrm{s} / \mathrm{n}$-Campus

Universitário. 36.570-000

Viçosa MG.

fadcco@gmail.com

\begin{abstract}
The main nutritional deficiencies during childhood, namely anemia and malnutrition, are predominantly related to socio-economic factors. Thus, as the Bolsa Familia Program (BFP) is the main policy to combat poverty, it is expected that it will have an impact on child nutrition. The aim was to analyze the differences in the nutritional situation of children registered with the BFP of a municipality located in Zona da Mata of Minas Gerais state. 446 children aged between 6 and 84 months were evaluated, of which 262 were non-beneficiaries and 184 were beneficiaries. Nutritional evaluation included analysis of weight and height parameters through weight/age, weight/height, height/age and Body Mass Index/ age indexes and hemoglobin levels, using the Hemocue. The prevalence of anemia, short stature and obesity were 22.6, 6.3 and 5.2\%, respectively, and there were no statistical differences between beneficiaries and non-beneficiaries. The beneficiary group initially had worse socio-economic conditions, but with the BFP it managed to financially match the non-beneficiary group. It is possible that the similarity between the two groups, also in the nutritional status, can be attributed to the program benefits, due to the financial funding as well as to the nutritional monitoring required as a condition of the program.
\end{abstract}

Key words Malnutrition, Anemia, Income, Poverty
Resumo Anemia e desnutrição, principais carências nutricionais na infância, têm como principais determinantes os socioeconômicos. Assim, por se tratar da principal politica de combate à pobreza, espera-se que o Programa Bolsa Família (PBF) promova impacto no estado nutricional infantil. Objetivou-se analisar as diferenças na situação nutricional de crianças cadastradas no PBF de um município da Zona da Mata Mineira. Foram avaliadas 446 crianças com idade entre $6 e$ 84 meses, sendo que 262 eram beneficiárias e 184 não-beneficiárias. A avaliação nutricional constituiu-se da análise dos parâmetros peso e estatura, através dos indices peso/idade, peso/estatura, estatura/idade e Índice de Massa Corporal/idade, $e$ dos niveis de hemoglobina, com uso do Hemocue. As prevalências de anemia, déficit estatural e obesidade foram 22,6, 6,3 e 5,2\%, respectivamente, sendo que não houve diferença estatística entre os beneficiários e não-beneficiários. Inicialmente, o grupo beneficiário apresentava piores condições socioeconômicas, porém, com o recebimento do benefício, os grupos se igualaram financeiramente. É possivel que a similaridade dos dois grupos também quanto ao estado nutricional possa ser atribuída ao recebimento do benefício, tanto devido ao incremento financeiro, quanto ao acompanhamento nutricional exigido como condicionalidade do programa.

Palavras-chave Desnutrição, Anemia, Renda, Pobreza 


\section{Introdução}

A cada ano, mais de 10 milhões de pessoas morrem devido à fome e à desnutrição. Destas, milhões também sofrem de deficiência de vitaminas e minerais, a chamada "fome oculta".

A anemia por deficiência de ferro e a desnutrição energético-proteica são as carências nutricionais mais comuns na infância. Este grupo populacional é mais susceptível ao desenvolvimento de deficiências nutricionais, devido ao rápido crescimento e à maior vulnerabilidade em que se encontram ${ }^{2}$. Estima-se que no Brasil a anemia afete aproximadamente $55 \%$ das crianças menores de cinco anos, e, no mundo, 47\%, sendo considerado um grave problema de saúde pública ${ }^{1,3}$.

Em relação à prevalência de déficits antropométricos, dados da última Pesquisa Nacional de Demografia e Saúde da Criança e da Mulher (PNDS), realizada em 2006, mostram que, entre as crianças menores de cinco anos, $7 \%$ se encontram com déficit estatural, $2 \%$ com déficit de peso para a estatura e 1,7\% com déficit de peso para a idade ${ }^{4}$. Crianças desnutridas têm pior rendimento escolar, dificuldades de aprendizagem, maior susceptibilidade a doenças e maior risco de mortalidade ${ }^{1}$.

Anemia e desnutrição são carências multifatoriais e têm como forte determinante, dentre outros, a situação socioeconômica, principalmente a renda. $\mathrm{O}$ baixo poder aquisitivo pode inviabilizar a compra de alimentos de melhor qualidade e em quantidade suficiente, podendo gerar assim uma situação de insegurança alimentar e de maior risco de deficiências nutricionais ${ }^{5}$. É importante ressaltar que o impacto exercido pela renda sobre estas carências se manifesta em diferentes intensidades, sendo a maior na desnutrição.

Partindo da premissa de que um incremento na situação financeira de uma família poderá promover uma melhora no estado nutricional das crianças que nela vivem, o governo brasileiro vem implantando, com êxito, programas de transferência de renda, como o Programa Bolsa Família (PBF).

O PBF é um programa de transferência direta de renda, criado em 2003, que visa reduzir a pobreza e as desigualdades e combater a fome entre as famílias pobres (com renda mensal per capita de $R \$ 60,01$ a $R \$ 120,00)$ e as extremamente pobres (com renda mensal per capita de até $\mathrm{R} \$$ 60,00). Para isso, pauta-se em três dimensões: promoção do alívio imediato da pobreza por meio da transferência direta de renda; reforço ao exercício de direitos sociais básicos nas áreas de Saúde e Educação; coordenação de programas complementares, para que as famílias consigam superar a situação de vulnerabilidade e pobreza ${ }^{6}$. Deste modo, espera-se que o beneficiário possa cumprir o exercício da cidadania e comportar-se como agente econômico, amparando não só a si e à sua família, mas inserindo-se num contexto mais amplo de relações por meio das quais poderá suavizar os efeitos de sua condição de pobreza ${ }^{7}$.

Este programa tem como contrapartida, o cumprimento de algumas condições pelas famílias beneficiadas, como a atualização do calendário vacinal e o acompanhamento mensal do crescimento para as menores de 7 anos, assim como o cumprimento da frequência escolar para crianças e adolescentes de 6 a 17 anos $^{6}$. A intenção é elevar o grau de concretização de direitos sociais por meio da indução da oferta e da demanda por serviços de saúde, educação e assistência social, e romper o ciclo da reprodução contínua da pobreza ${ }^{8}$.

Ressalta-se que alguns trabalhos publicados visando avaliar a influência dos programas de transferência de renda sobre a saúde infantil não confirmaram um impacto positivo ou então foram pouco conclusivos ${ }^{9-11}$. Frente a essa questão, os estudos com esta finalidade têm fundamental importância, tanto devido ao elevado investimento do governo em programas sociais, com ênfase no PBF, quanto à elevada expectativa da população na melhoria das condições de vida e saúde, possivelmente viabilizadas através do recebimento destes benefícios.

Neste sentido, o objetivo deste estudo foi analisar as diferenças na situação nutricional de crianças beneficiárias e não-beneficiárias residentes em um município de pequeno porte da Zona da Mata Mineira, com ênfase no perfil antropométrico e níveis de hemoglobina.

\section{Metodologia}

O presente trabalho foi realizado no município de Paula Cândido, localizado na Zona da Mata de Minas Gerais, durante os meses de setembro a novembro de 2007. De acordo com o Instituto Brasileiro de Geografia e Estatística (IBGE), trata-se de um município com 9.109 habitantes, cuja extensão territorial é de $269 \mathrm{~km}^{2}$, e $57 \%$ da população reside na zona rural. A proporção de crianças é $19,4 \%$, sendo que $13 \%$ são menores de sete anos.

Aproximadamente $30 \%$ da população não possui nenhum rendimento nominal, e $8 \%$ pos- 
sui menos de um ano de estudo ${ }^{12}$. O Índice de Gini é de 0,42, a incidência da pobreza, 32,94\% e o Índice de Desenvolvimento Humano (IDH) é de 0,699 , considerado médio ${ }^{12,13}$.

Trata-se de um estudo transversal, no qual foram avaliadas 446 crianças cadastradas no PBF (69,1\% do total de crianças cadastradas e 37,3\% do total de crianças menores de sete anos residentes no município), com idade entre 6 e 84 meses, pertencentes a dois grupos distintos: o que recebe o benefício (grupo BF) e aquele cadastrado no programa mas que não recebe (grupo NBF). A amostra final foi composta por 184 crianças do grupo NBF (87,6\% do total de crianças cadastradas não-beneficiadas), e 262 crianças do grupo BF $(60,2 \%$ do total de crianças cadastradas beneficiadas), sendo que estas foram selecionadas através de amostragem probabilística.

A faixa etária de até 84 meses foi fixada com base na idade na qual a criança realizava o acompanhamento nutricional, como uma das condicionalidades do PBF. As crianças menores de seis meses de idade foram excluídas da seleção devido à inexistência de pontos de corte recomendados internacionalmente para diagnóstico de anemia.

O teste de anemia foi realizado através de punção digital no dedo anular da mão não-dominante em crianças que já deambulavam, e por punção no calcanhar, nas que ainda não o faziam, ambos retirando apenas uma gota de sangue $^{14}$. O equipamento utilizado para a determinação dos níveis de hemoglobina foi o hemoglobinômetro portátil Hemocue ${ }^{\circledR}$, e os pontos de corte adotados para caracterizar anemia foram os preconizados pela OMS, de 11,0 g/dL para crianças de 6 a 59 meses e 11,5 g/dL para crianças e adolescentes de 5 a 11 anos15. Para determinar a gravidade da anemia, foram utilizados os seguintes pontos de cortes: menor que 7,0 g/dL para anemia grave; de 7,0 a 9,9 g/dL para anemia moderada, de 10,0 a 10,9 g/dL para anemia leve em crianças de 6 a 59 meses, e 10,0 a 11,4 g/dL para anemia leve em crianças e adolescentes de 5 a 11 $\operatorname{anos}^{15}$.

Os parâmetros antropométricos aferidos foram peso e estatura/comprimento, coletados por nutricionista treinada, baseando-se nas técnicas recomendadas por Jelliffe ${ }^{16}$. Para a classificação do estado nutricional utilizou-se a referência internacional da OMS, de $2006^{17}$. Os dados de peso e estatura/comprimento foram convertidos em escore-Z, e os índices utilizados foram Peso/Idade (P/I), Peso/Estatura (P/E), Estatura/Idade (E/ I) e Índice de Massa Corporal/Idade (IMC/I).
Para as crianças maiores de cinco anos não se utilizou o índice $\mathrm{P} / \mathrm{E}$ por não ser recomendado pela OMS para esta faixa etária. Os pontos de corte adotados para classificação de baixo peso e sobrepeso para os índices P/E, P/I e IMC/I foram -2.00 e +2.00 escores $-Z$, respectivamente. O ponto de corte de -2.00 escores- $Z$ foi também utilizado para diagnóstico de baixa estatura ${ }^{18}$.

Foram aplicados questionários semi-estruturados com os pais ou responsáveis, os quais continham questões a respeito da saúde da criança e de sua família, e também questões abordando as características socioeconômicas. Estes questionários foram elaborados pela equipe de pesquisadores responsáveis pelo projeto a partir de levantamento na literatura científica.

A análise de homogeneidade de variância foi empregada aplicando-se o teste de Bartlett. Para verificar a associação entre variáveis categóricas utilizou-se o teste do Qui-quadrado de Pearson (c2 de Pearson) e, no caso da análise de três ou mais variáveis, quando este teste apresentou significância estatística, foi realizada a sua partição. Para comparação de dois grupos independentes, considerando as variáveis numéricas, foi utilizado o teste $t$ de Student ou Mann-Whitney, de acordo com a distribuição da variável. O coeficiente de correlação de Pearson foi utilizado para testar a correlação linear entre o tempo de recebimento do benefício e os valores de escore- $Z$ e de hemoglobina. O nível de significância adotado em todos os testes para a rejeição da hipótese de nulidade foi inferior a 5\%.

Os softwares utilizados para confecção do banco de dados e análises estatísticas foram o Epi Info, versão 6.04, e o Sigma, versão 2.03. O estado nutricional das crianças foi avaliado com o auxílio do software WHO Anthro (2005).

Todas as crianças avaliadas tiveram a autorização dos pais ou responsáveis, mediante assinatura do termo de consentimento livre e esclarecido. Aquelas detectadas como anêmicas foram encaminhadas para o médico da respectiva Equipe de Saúde da Família para iniciar o tratamento. Todo medicamento necessário para a respectiva intervenção foi adquirido pela Prefeitura Municipal de Paula Cândido, através do Sistema Único de Saúde.

As crianças distróficas foram encaminhadas para a nutricionista da rede básica. O projeto foi previamente aprovado pelo Comitê de Ética em Pesquisas com Seres Humanos da Universidade Federal de Viçosa, em conformidade com a Resolução 196/96 do Conselho Nacional de Saúde ${ }^{19}$. 


\section{Resultados}

Das 446 crianças avaliadas, $51,8 \%$ era do sexo masculino, a média e a mediana de idade foram 46,41 meses ( $\pm 22,18$ meses) e 47 meses (mínimo de 6 e máximo de 84 meses), respectivamente.

Dentre as crianças avaliadas, $384(86 \%)$ tiveram sua renda informada pelo responsável. $\mathrm{Na}$ Tabela 1 estão evidenciados os valores de renda familiar e per capita, incluindo-se ou não os valores do benefício. Observa-se que, sem o valor do benefício, o grupo NBF apresentou rendas familiar e per capita significantemente maiores do que o grupo BF. O fato da escolaridade dos pais ter sido significantemente maior no grupo NBF pode contribuir para justificar a maior renda observada neste grupo. Ao incluir o valor do benefício à renda, verificou-se que o grupo BF passa a ter renda familiar significantemente maior, e se iguala estatisticamente ao grupo NBF em relação à renda per capita.

O número de crianças ou adolescentes presentes no domicílio, e consequentemente o número de pessoas residentes no mesmo, foi significantemente maior no grupo BF, o que já era esperado uma vez que famílias numerosas são mais vulneráveis, e assim, são priorizadas no recebimento do benefício.

As profissões paternas mais relatadas foram as de lavrador, pedreiro, servente de pedreiro e ordenhador, sendo que o grupo BF possuía mais pais lavradores do que o NBF (61,8\% x 59,5\%). Entre as profissões maternas, as mais relatadas foram donas de casa, lavradoras e domésticas/ faxineiras, sendo que no grupo BF havia mais mães donas de casa do que no NBF $(65,5 \% \mathrm{x}$ $59,9 \%)$. No grupo BF, seis pais $(2,7 \%)$ e três mães $(1,2 \%)$ não completaram o primeiro ano de estudo. No grupo NBF, dois pais $(1,3 \%)$ e nenhuma mãe se encontravam nesta situação.

Em relação às características relativas ao saneamento básico, o grupo NBF apresentou maior percentual de lixo coletado $(55,5 \%$ x $46,2 \%)$ e de fornecimento de água $(53,3 \%$ x $43,9 \%)$ e esgoto $(67,4 \%$ x 69,0\%) pela rede pública, assim como tratamento da água por filtração $(77,4 \% \times 76,2 \%)$, porém essas diferenças não foram significantes.

Não houve diferença estatística entre os grupos BF e NBF em relação à frequência de anemia (Tabela 2), bem como em relação ao estado nutricional (Tabelas 3 e 4). Ao comparar os níveis médios de hemoglobina, observou-se que não

Tabela 1. Caracterização socioeconômica das famílias cadastradas no Programa Bolsa Família, segundo recebimento ou não do benefício. Paula Cândido, MG, 2007.

\begin{tabular}{|c|c|c|c|c|c|}
\hline \multirow[b]{2}{*}{ Características } & \multicolumn{2}{|c|}{ Grupo BF ${ }^{1}$} & \multicolumn{2}{|c|}{ Grupo NBF $^{2}$} & \multirow[b]{2}{*}{$\mathbf{p}$} \\
\hline & $\mathrm{X} \pm \mathrm{DP}$ & Med (mín-máx) & $\mathrm{X} \pm \mathrm{DP}$ & Med (mín-máx) & \\
\hline $\begin{array}{l}\text { Renda familiar, excluindo } \\
\text { o benefício (em reais) }\end{array}$ & $335,10 \pm 139,40$ & $\begin{array}{c}380,00 \\
(0,00-1040,00)\end{array}$ & $391,40 \pm 245,00$ & $\begin{array}{c}380,00 \\
(60,00-2000,00)\end{array}$ & $0,01^{\text {a }}$ \\
\hline $\begin{array}{l}\text { Renda familiar, incluindo o } \\
\text { benefício (em reais) }\end{array}$ & $413,96 \pm 136,66$ & $\begin{array}{c}416,00 \\
(50,00-1055,00)\end{array}$ & $391,40 \pm 245,00$ & $\begin{array}{c}380,00 \\
(60,00-2000,00)\end{array}$ & $<0,01^{a}$ \\
\hline $\begin{array}{l}\text { Renda per capita, } \\
\text { excluindo o benefício } \\
\text { (em reais) }\end{array}$ & $69,60 \pm 31,31$ & $\begin{array}{c}63,30 \\
(0,00-190,00)\end{array}$ & $94,70 \pm 72,00$ & $\begin{array}{c}85,70 \\
(14,00-666,70)\end{array}$ & $<0,01^{a}$ \\
\hline $\begin{array}{l}\text { Renda per capita, } \\
\text { incluindo o benefício } \\
\text { (em reais) }\end{array}$ & $85,38 \pm 30,14$ & $\begin{array}{c}82,40 \\
(16,67-197,50)\end{array}$ & $94,70 \pm 72,00$ & $\begin{array}{c}85,70 \\
(14,00-666,60)\end{array}$ & $0,94^{\mathrm{a}}$ \\
\hline $\begin{array}{l}\mathrm{N}^{\circ} \text { de residentes no } \\
\text { domicílio }\end{array}$ & $5,0 \pm 1,5$ & $\begin{array}{c}5 \\
(2-11)\end{array}$ & $4,6 \pm 2,0$ & $\begin{array}{c}4 \\
(2-15)\end{array}$ & $<0,01^{a}$ \\
\hline $\begin{array}{l}\text { No de crianças e } \\
\text { adolescente (menores que } \\
15 \text { anos) }\end{array}$ & $2,7 \pm 1,3$ & $\begin{array}{c}3 \\
(1-8)\end{array}$ & $2,1 \pm 1,1$ & $\begin{array}{c}2 \\
(1-7)\end{array}$ & $<0,01^{\text {b }}$ \\
\hline $\begin{array}{l}\text { Escolaridade paterna } \\
\text { (em anos de estudo) }\end{array}$ & $4,4 \pm 2,4$ & $\begin{array}{c}4 \\
(0-11)\end{array}$ & $5,2 \pm 2,7$ & $\begin{array}{c}4 \\
(0-15)\end{array}$ & $0,002^{\mathrm{b}}$ \\
\hline $\begin{array}{l}\text { Escolaridade materna } \\
\text { (em anos de estudo) }\end{array}$ & $4,9 \pm 2,4$ & $\begin{array}{c}4 \\
(0-11)\end{array}$ & $5,7 \pm 2,7$ & $\begin{array}{c}5 \\
(1-13)\end{array}$ & $0,005^{\mathrm{a}}$ \\
\hline
\end{tabular}

$\mathrm{X} \pm \mathrm{DP}=$ média \pm desvio padrão; Med = mediana; Mín = mínimo; Máx = máximo; ${ }^{\mathrm{a}}$ Mann - Whitney; ${ }^{\mathrm{b}}$ Teste $\mathrm{t}$ de Student; 1 = Grupo beneficiário; $2=$ Grupo não-beneficiário; Nota $=n$ pode diferir discretamente entre as variáveis devido a perdas. 
Tabela 2. Frequência de anemia em crianças de 6 a 84 meses cadastradas no Programa Bolsa Família, segundo recebimento ou não do benefício. Paula Cândido, MG, 2007.

\begin{tabular}{|c|c|c|c|c|c|c|}
\hline & \multicolumn{2}{|c|}{ Todos ${ }^{1}$} & \multicolumn{2}{|c|}{ Grupo BF ${ }^{2}$} & \multicolumn{2}{|c|}{ Grupo NBF } \\
\hline & $\mathbf{n}$ & $\%$ & $\mathbf{n}$ & $\%$ & $\mathbf{n}$ & $\%$ \\
\hline Anemia ${ }^{a}$ & 100 & 22,6 & 57 & 22,0 & 43 & 23,4 \\
\hline Anemia leve ${ }^{b}$ & 69 & 69,0 & 39 & 68,4 & 30 & 69,8 \\
\hline Anemia moderada b & 30 & 30,0 & 17 & 29,8 & 13 & 30,2 \\
\hline Anemia grave ${ }^{b}$ & 1 & 1,0 & 1 & 1,8 & - & - \\
\hline
\end{tabular}

${ }^{a} \mathrm{p}>0,05$ no teste do $\chi^{2}$ entre anêmicos e não anêmicos $\mathrm{x}$ grupos BF e NBF; ${ }^{\mathrm{b}} \mathrm{p}>0,05$ no teste do $\chi^{2}$ entre anemia leve e anemia moderada+grave $\mathrm{x}$ grupos $\mathrm{BF}$ e NBF.

$1=$ Cadastrados $(n=443) ; 2=$ Grupo beneficiário $(n=259)$;

3= Grupo não-beneficiário $(\mathrm{n}=184)$. houve diferença significante entre os grupos $(\mathrm{BF}=$ $11,94+-1,25 \mathrm{~g} / \mathrm{dL} ; \mathrm{NBF}=11,79+-1,13 \mathrm{~g} / \mathrm{dL})$.

Estratificando-se a amostra de acordo com as faixas etárias de maior risco para anemia, também não foi verificada diferença estatística entre a frequência de anemia ou níveis médios de hemoglobina e os grupos BF e NBF (dados não apresentados). Esta análise também foi aplicada entre as médias de escore-Z para os índices $\mathrm{P} / \mathrm{E}, \mathrm{E} / \mathrm{I}, \mathrm{P} /$ I e IMC/I e os grupos BF e NBF, porém não houve diferença estatística (dados não apresentados).

Embora sem diferença estatística, o grupo BF apresentou, quando comparado ao NBF, percentual ligeiramente superior de baixo peso para idade e baixa estatura (Tabela 4). No entanto, dentre as crianças com desnutrição crônica (E/I e $\mathrm{P} / \mathrm{I}$ inferior a -2.00 escores-Z), as pertencentes ao

Tabela 3. Medidas de tendência central e de variabilidade dos valores de escores- $Z$ das crianças cadastradas no Programa Bolsa Família, segundo recebimento ou não do benefício. Paula Cândido, MG, 2007.

\begin{tabular}{|c|c|c|c|c|c|c|}
\hline \multirow[b]{2}{*}{ Índices } & \multicolumn{2}{|c|}{ Todos $^{1}$} & \multicolumn{2}{|c|}{ Grupo $\mathrm{BF}^{2}$} & \multicolumn{2}{|c|}{ Grupo $\mathrm{NBF}^{3}$} \\
\hline & $\mathrm{X} \pm \mathrm{DP}$ & Med (mín-máx) & $\mathrm{X} \pm \mathrm{DP}$ & Med (mín-máx) & $\mathrm{X} \pm \mathrm{DP}$ & Med (mín-máx) \\
\hline $\mathrm{P} / \mathrm{I}$ & $-0,04 \pm 1,08$ & $\begin{array}{c}-0,20 \\
(-3,50-7,10)\end{array}$ & $-0,09 \pm 1,10$ & $\begin{array}{c}-0,20 \\
(-2,30-7,10)\end{array}$ & $0,02 \pm 1,04$ & $\begin{array}{c}0,05 \\
(-3,50-3,70)\end{array}$ \\
\hline $\mathrm{P} / \mathrm{E}^{*}$ & $0,31 \pm 1,08$ & $\begin{array}{c}0,30 \\
(-2,30-5,20)\end{array}$ & $0,24 \pm 1,05$ & $\begin{array}{c}0,20 \\
(-2,00-5,20)\end{array}$ & $\begin{array}{r}0,40 \pm 1,12 \\
-\end{array}$ & $\begin{array}{c}0,40 \\
(-2,30-4,20)\end{array}$ \\
\hline $\mathrm{E} / \mathrm{I}$ & $-0,40 \pm 1,04$ & $\begin{array}{c}-0,40 \\
(-4,70-3,40)\end{array}$ & $-0,41 \pm 1,09$ & $\begin{array}{c}-0,40 \\
(-4,70-3,40)\end{array}$ & $0,38 \pm 0,98$ & $\begin{array}{c}-0,30 \\
(-3,70-2,00)\end{array}$ \\
\hline IMC/I & $0,27 \pm 1,13$ & $\begin{array}{c}0,10 \\
(-2,20-6,50)\end{array}$ & $0,21 \pm 1,13$ & $\begin{array}{c}0,10 \\
(-2,00-6,50)\end{array}$ & $0,36 \pm 1,12$ & $\begin{array}{c}0,30 \\
(-2,20-4,30)\end{array}$ \\
\hline
\end{tabular}

$\mathrm{X} \pm \mathrm{DP}=$ média \pm desvio padrão; Med = mediana; Mín = mínimo; Máx = máximo. $1=$ Cadastrados; $2=$ Grupo beneficiário; $3=$ Grupo não-beneficiário. ${ }^{*}=$ crianças até 60 meses. Nota $=n$ pode diferir discretamente entre as variáveis devido a perdas. p $>0,05$ no teste $t$ de Student entre as médias de escore-Z e os grupos BF e NBF.

Tabela 4. Frequência de distúrbios nutricionais em crianças cadastradas no Programa Bolsa Família, segundo recebimento ou não do benefício. Paula Cândido, MG, 2007.

\begin{tabular}{|c|c|c|c|c|c|c|}
\hline \multirow[b]{2}{*}{ Classificação } & \multicolumn{2}{|c|}{ Todos ${ }^{1}$} & \multicolumn{2}{|c|}{ Grupo BF $^{2}$} & \multicolumn{2}{|c|}{ Grupo NBF ${ }^{3}$} \\
\hline & $\mathbf{n}$ & $\%$ & $\mathbf{n}$ & $\%$ & $\mathbf{n}$ & $\%$ \\
\hline Peso baixo para idade & 9 & 2,0 & 6 & 2,3 & 3 & 1,6 \\
\hline Peso baixo para estatura* & 3 & 1,0 & - & - & 3 & 2,2 \\
\hline Baixo IMC para idade & 2 & 0,5 & 1 & 0,4 & 1 & 0,5 \\
\hline Baixa estatura para idade & 28 & 6,3 & 17 & 6,5 & 11 & 6,0 \\
\hline Peso elevado para idade & 17 & 3,8 & 9 & 3,4 & 8 & 4,3 \\
\hline Peso elevado para estatura* & 13 & 2,9 & 6 & 3,6 & 7 & 5,2 \\
\hline Obesidade (IMC/idade) & 23 & 5,2 & 11 & 4,2 & 12 & 6,6 \\
\hline
\end{tabular}

p $>0,05$ no teste do $\chi^{2}$ entre os grupos BF e NBF. $1=$ Cadastrados; 2 = Grupo beneficiário; 3 = Grupo não-beneficiário.

* = crianças até 60 meses 
grupo BF tiveram maior média de escore-Z, tanto para o índice $\mathrm{P} / \mathrm{I}$ quanto para o $\mathrm{E} / \mathrm{I}$, do que as do NBF, sem diferença estatística (dados não apresentados). Nota-se ainda que todas as crianças identificadas com peso baixo para estatura estavam no grupo NBF.

Dentre as 226 mães que souberam relatar a quanto tempo recebiam o benefício, $48,2 \%$ disseram receber a um ano ou menos, 32,3\% entre um e dois anos e 19,5\% a três anos ou mais. O tempo mediano de recebimento foi de 15 meses (mínimo de 1 e máximo de 96 meses). Ao comparar o estado nutricional e os níveis de hemoglobina das crianças em função do tempo de recebimento do benefício, não houve diferença entre o grupo que recebia a mais ou menos de 15 meses. Há que se ressaltar que as crianças com déficits antropométricos e anemia recebiam o benefício há menos tempo que as sem déficits e não anêmicas, porém essa diferença não foi significativa.

Também não foi verificada correlação entre o tempo de recebimento do benefício e os níveis de hemoglobina $(r=0,03 ; p=0,557)$, ou o estado nutricional para os índices IMC/I $(\mathrm{r}=-0,02 ; \mathrm{p}=$ $0,691), E / I(r=-0,02 ; p=0,662), P / I(r=-0,03 ; p=$ $0,604)$ e $\mathrm{P} / \mathrm{E}(\mathrm{r}=0,06 ; \mathrm{p}=0,448)$.

Analisando o cumprimento das condicionalidades, verificou-se que 9,2\% das crianças beneficiárias (24 crianças) não realizavam o acompanhamento antropométrico mensalmente, sete $(2,7 \%)$ não estavam com a vacinação completa e $10,9 \%$ ainda não estavam matriculadas na escola.

Não houve diferença estatística entre os níveis de hemoglobina ou médias de escore-Z entre as que estavam com a vacinação completa e as que não estavam, nem entre as que realizavam acompanhamento antropométrico ou não. Também não houve diferença entre os parâmetros socioeconômicos nos grupos que cumpriam ou não as condicionalidades.

\section{Discussão}

Analisando a variável renda, sem incluir o valor do benefício, verificou-se que o grupo NBF apresentou rendas familiar e per capita estatisticamente superiores ao grupo BF, o que foi atribuído a uma possível realização de cadastros fora do perfil. Um dos critérios de inclusão no PBF é que a renda mensal da família seja inferior a 120 reais per capita. Neste estudo, 51 famílias apresentaram renda per capita superior a este valor, sendo que 19 (37,3\%) eram do grupo BF, ou seja, recebiam o benefício sem se encaixarem no perfil da seleção. Isto aponta para o fato de que o município estudado ainda apresenta problemas operacionais, que podem ser reflexos das dificuldades referentes à implantação. Segundo Fonseca e Roquete $^{20}$, as prefeituras necessitavam de regras claras para desenvolverem um bom trabalho de cadastramento, além da rigorosa fiscalização do processo pelas instâncias competentes. A exclusão das famílias fora do perfil não alterou os resultados aqui apresentados.

Não obstante, no presente estudo, através da comprovação de melhores condições sociossanitárias no grupo NBF, avaliados aqui em termos de renda, escolaridade e condições de saneamento, constata-se que, de maneira geral, o PBF tem, de fato, focalizado famílias socialmente mais vulneráveis, dentro de um grupo já considerado vulnerável, que é o de famílias cadastradas no programa. Assim, conclui-se que estão cumprindo seu objetivo.

A prevalência de anemia entre as 443 crianças avaliadas no município de Paula Cândido $(22,6 \%)$ foi relativamente baixa, possivelmente devido à ampla faixa etária estudada, na qual $78,5 \%$ das crianças eram maiores de dois anos de idade. A maioria das crianças anêmicas (69\%) apresentou um quadro leve. Avaliando apenas as menores de cinco anos, a prevalência de anemia foi de $25,8 \%$, e entre as menores de dois anos, foi de 44,9\%. Dessa forma, é possível concluir que a prevalência da anemia no caso presente, independente da faixa etária avaliada, é inferior a da maioria dos estudos brasileiros.

É importante ressaltar que, pelo fato deste estudo contemplar um grupo específico de crianças, apenas as cadastradas no PBF, as prevalências aqui encontradas não podem ser extrapoladas para as brasileiras como um todo, mas indicam como podem se comportar as pertencentes a programas de transferência de renda de municípios, com as mesmas características do aqui estudado. Imaginando que $22,0 \%$ das 7.380 .009 crianças brasileiras beneficiárias, menores de sete anos, que têm acompanhamento de seu estado nutricional ${ }^{21}$ estejam anêmicas, tem-se uma estimativa de que aproximadamente 1.623 .601 crianças beneficiárias do PBF possam estar anêmicas. Diante da magnitude desta deficiência, evidenciada nesta análise, fica clara a importância de priorizar o combate à anemia na agenda de saúde de todo o Brasil, e até mesmo incluir o acompanhamento desta carência na vigilância do estado nutricional das crianças beneficiárias.

Em relação aos dados antropométricos, encontrou-se neste estudo uma prevalência de 6,3\% 
de baixa estatura, 2,0\% de baixo peso para idade, $1,0 \%$ de baixo peso para estatura, $0,5 \%$ de baixo IMC para idade, $3,8 \%$ de peso elevado para a idade, 2,9\% de peso elevado para estatura e 5,2\% de obesidade. Com isso, confirma-se que a baixa estatura e o excesso de peso aparecem como os principais problemas nutricionais deste grupo, uma vez que obtiveram frequência acima do esperado para uma população com distribuição normal $(2,28 \%)$, corroborando com outros autores e com as últimas pesquisas nacionais ${ }^{22,23}$.

Segundo o acompanhamento registrado pelo Sistema de Vigilância Alimentar e Nutricional (SISVAN) até junho de 2008, dentre as crianças menores de cinco anos beneficiárias do PBF em todo o estado de Minas Gerais, 5,6\% apresentavam baixo peso para idade (entre os percentis $0,1$ e 3$), 6,7 \%$ apresentavam baixo peso para estatura e 12,5\% apresentavam baixa estatura para a idade. Encontravam-se acima do percentil 97, $10,5 \%$ das crianças pelo índice peso para idade, e 9,9\% pelo índice peso para estatura ${ }^{21}$. A título de comparação com os dados do SISVAN, os resultados do presente estudo foram convertidos em percentis e analisados apenas para os menores de cinco anos. Assim, as prevalências de déficits para os índices P/E, P/I e E/I foram de 2,0\%, 3,0\% e $8,6 \%$, respectivamente, e de excesso de peso para a estatura e para a idade, foram 5,6\% e 4,0\%, respectivamente. Apesar das diferentes referências antropométricas utilizadas nos estudos, notase que as prevalências publicadas pelo SISVAN, tanto para o excesso quanto para déficits nutricionais, estão muito superiores aos últimos dados nacionais e aos achados do presente estudo.

Um questionamento frequente é o de que o PBF utiliza como único critério de inclusão a ren$\mathrm{da}$, em detrimento de outros igualmente importantes, como por exemplo, o biológico, especificamente o estado nutricional. O critério socioeconômico também é utilizado para o repasse dos benefícios, priorizando as famílias com renda menor e maior número de pessoas vivendo no domicílio, principalmente mais crianças. Buscando um esclarecimento para esta questão, foram realizadas algumas análises entre as crianças desnutridas dos grupos BF e NBF. Verificou-se que o grupo NBF apresentou menos crianças desnutridas, porém estas foram as que apresentaram os piores valores de escore-Z. Esta análise conduz a duas vertentes de pensamento, que não puderam ser desvendadas por completo no presente estudo, pois não se sabe a ordem de causalidade entre as duas. Na primeira, supõe-se que o PBF pode estar falhando ao utilizar como cri- tério de transferência de benefícios apenas a renda, pois as crianças que não o recebem podem ter melhores condições socioeconômicas mas pior situação nutricional. Na segunda, imaginase que as crianças do grupo NBF estejam com piores graus de desnutrição por não terem a obrigatoriedade do acompanhamento nutricional, nem o benefício para incrementar a alimentação.

Não foi observada diferença entre os grupos BF e NBF em relação ao estado nutricional, níveis de hemoglobina e frequência de anemia, indo ao encontro dos resultados encontrados em estudo nacional realizado pelo Ministério da Saúde, o qual avaliou o impacto do Programa Bolsa Alimentação sobre o estado nutricional de crianças menores de sete anos ${ }^{10}$. Neste trabalho, a prevalência de baixa estatura para idade nas crianças beneficiárias foi de $15,1 \%$, a de baixo peso para a idade e a de baixo peso para a estatura foram de $10,7 \%$ e de $2,2 \%$, respectivamente, todos superiores aos achados do presente estudo. Os autores verificaram ainda que o grupo de crianças beneficiárias obteve maiores taxas de déficits nutricionais do que o grupo não beneficiário, sendo que para o índice E/I esta diferença foi significativa $^{10}$.

Ainda avaliando o Programa Bolsa Alimentação, resultados semelhantes foram encontrados em estudo nacional realizado por Morris et al. ${ }^{9}$, com crianças menores de sete anos. Estes autores observaram que, entre as beneficiárias, as prevalências de déficit de peso para idade e peso para estatura foram $9,9 \%$ e $1,8 \%$, respectivamente, e de baixa estatura, 14,3\%, sem diferença entre os grupos. Relataram ainda que as crianças menores de três anos, que recebiam o benefício, apresentaram menor ganho de peso do que as que não recebiam, fato que foi atribuído a um possível receio das mães em perder o benefício se as crianças melhorassem seu estado nutricional ${ }^{9}$.

É importante lembrar que o grupo NBF apresentava melhores condições de renda e escolaridade em relação ao grupo BF, sem contar com o valor do benefício recebido.

Quando este valor foi incluído nos montantes de renda familiar e per capita, o grupo BF passou a ter maior renda familiar e ambos se igualaram estatisticamente em termos de renda per capita. Como não houve diferença estatística entre os grupos em termos de estado nutricional, uma hipótese seria sugerir que o benefício recebido pelo grupo BF possa estar contribuindo para a manutenção da semelhança encontrada entre os parâmetros nutricionais nos dois gru- 
pos, tanto devido ao incremento financeiro que possivelmente melhoraria a alimentação, quanto à obrigatoriedade do acompanhamento nutricional. Porém, como se trata de uma análise transversal, é difícil concluir com segurança se esta diferença representa o efeito do programa ou, se simplesmente a persistência de diferenças já existentes antes do PBF ter início ${ }^{10}$.

Para fundamentar a hipótese de que o benefício possa estar contribuindo na melhoria do estado nutricional das crianças, Magalhães et al. ${ }^{24}$ realizaram um estudo com a mesma população, e verificaram que a maioria das famílias entrevistadas $(76,4 \%)$ gastava o benefício com alimentação. Em relatório publicado pelo Instituto Brasileiro de Análises Sociais e Econômicas (IBASE), também foi constatado o mesmo destino para o recurso do PBF na maioria das famílias brasileiras $(87,0 \%)$. Muitas delas aumentavam a quantidade $(73,7 \%)$ ou a variedade $(69,8 \%)$ de alimentos consumidos, e outras a aquisição de alimentos de preferência da criança (62,8\%). Aproximadamente $61,0 \%$ das famílias consumiram mais carne $\mathrm{e}^{25}$.

A Comissão Nacional dos Determinantes Sociais em Saúde avalia que os achados atuais sobre o impacto do PBF no estado nutricional infantil ainda são inconclusivos, embora esteja claro que o benefício tenha contribuído para a redução da desigualdade social brasileira e para a ampliação da variedade de alimentos consumidos pelas famílias beneficiárias ${ }^{11}$.

É necessário destacar que, apesar das famílias possivelmente estarem utilizando o benefício para a aquisição de alimentos, outros fatores, além da melhoria na alimentação, podem contribuir para que tenha havido algum impacto no estado nutricional, tais como acesso aos serviços de saúde, saneamento básico e, principalmente, o tempo de recebimento do benefício ${ }^{25}$.

Neste estudo foi avaliado se o tempo de recebimento do benefício do PBF, relatado pelas mães, exercia alguma influência sobre os níveis de hemoglobina ou os valores de escore- $Z$ das crianças cadastradas, o que não ocorreu. A maioria destas recebia o benefício há um ano, o que remete à reflexão se este tempo seria suficiente para que, através de uma possível melhora na alimentação, haja modificações nos parâmetros de hemoglobina, peso e estatura. Responder a este questionamento pode ser objeto para futuros estudos, pois sabe-se que, se o incremento na dieta ocorrer de fato, diversos outros fatores poderão influenciar negativa ou positivamente, como a qualidade nutricional da dieta, inclusive em termos de micronutrientes, a presença de doenças infecciosas e parasitárias e as variáveis ambientais.

Verificou-se que a maioria das crianças que não realizavam o acompanhamento antropométrico mensal e que não frequentavam a escola residia em área rural, o que leva a crer que os principais obstáculos para essas famílias sejam a inacessibilidade geográfica ou a inexistência de serviços disponíveis nestas comunidades. O cumprimento dos objetivos do PBF pode ser interpretado pelas famílias beneficiárias como a materialização do direito à saúde e à educação, contribuindo assim para redução da pobreza. Para o poder público, pode estimular o aumento da oferta local de serviços de saúde, educação e assistência social, e identificar as famílias em situação de maior vulnerabilidade social, para que sejam encaminhadas a ações específicas de acompanhamento ${ }^{8}$. No entanto, é importante ressaltar que o cumprimento é dever tanto das famílias como do governo, pois se os serviços não estiverem acessíveis à população, principalmente a residente em área rural, torna-se inviável sua realização por parte das famílias.

\section{Considerações finais}

Pode-se dizer que entre os cadastrados no PBF a prevalência de anemia é um problema de saúde pública de grau moderado, e juntamente com a baixa estatura e o excesso de peso, são distúrbios nutricionais que merecem especial atenção por parte do sistema de saúde.

Evidenciou-se neste trabalho problemas de falta de fiscalização no repasse dos benefícios, uma vez que famílias fora do perfil exigido pelo programa eram beneficiadas. Apesar disso, parece que o PBF atinge, de fato, as famílias mais necessitadas.

Propõe-se que o município dirija especial atenção ao controle social e aos comitês gestores, a fim de melhorar a operacionalização do programa.

As melhores condições socioeconômicas no grupo NBF e a semelhança estatística na avaliação nutricional entre os grupos BF e NBF sugerem que o benefício foi importante aqui, tanto pelo aporte financeiro que pode estar incrementando a alimentação, quanto pela obrigatoriedade do acompanhamento nutricional exigido.

É evidente a influência dos fatores socioeconômicos dos pais, como a escolaridade, sobre a saúde dos filhos, gerando um ciclo de pobreza que se mantém por gerações. Sob este ponto de 
vista, o PBF tem como pontos positivos a obrigatoriedade do cumprimento das exigências que visam quebrar este ciclo através da melhoria da educação e saúde das crianças e adolescentes, e a criação de programas complementares, visando à autonomia das famílias. Para isso, é importante que, além da evidência científica, a mobilização e a participação popular, assim como a dos profissionais de saúde, sejam os pilares de sustentação da solução das iniquidades.

\section{Colaboradores}

FCC Oliveira foi responsável pela concepção do projeto, coleta e análise dos dados e redação do manuscrito. SCC Franceschini colaborou em todas as etapas do estudo, incluindo concepção, análise dos dados, interpretação dos resultados e revisão do manuscrito. RMM Cotta, SE Priore e LFR Sant'Ana contribuíram na concepção do estudo e revisão do manuscrito.

\section{Agradecimentos}

Ao CNPq e à FAPEMIG pelo financiamento do projeto. À Prefeitura Municipal de Paula Cândido, em especial à nutricionista da rede básica, Karine Oliveira Gomes, pelo apoio.

\section{Referências}

1. McLean E, Cogswell M, Egli I, Wojdyla D, Benoist B. Worldwide prevalence of anemia in preschool aged children, pregnant women and non-pregnant women of reproductive age. In: Kraemer K, Zimmermann MB. Nutritional Anemia. Switzerland: Sight and Life Press; 2007. p. 1-12.

2. Biscegli TS, Correa CEC, Romera J, Hernandez JLJ. Avaliação do estado nutricional e prevalência da carência de ferro em crianças frequentadoras de uma creche. Rev Paul Pediatr 2006; 24(4):323-329.

3. World Health Organization (WHO). Department of Health \& Human Services - USA. Center for Disease Control and Prevention (CDC). Worldwide prevalence of anaemia 1993-2005. WHO Global Database on Anaemia. Geneva; 2008.

4. Brasil. Ministério da Saúde. PNDS 2006. Pesquisa Nacional de Demografia e Saúde da Criança e da Mulher. Brasília, 2008 [acessado 2008 jul 15]. Disponível em: http://www.saude.gov.br/pnds2006

5. Souto TS, Oliveira MN, Casoy F, Machado EHS, Juliano Y, Gouvêa LC, Armond JE. Anemia e renda per capita familiar de crianças frequentadoras da creche do Centro Educacional Unificado Cidade Dutra, no Município de São Paulo. Rev Paul Pediatria 2007; 25(2):161-166.

6. Brasil. Ministério do Desenvolvimento Social e Combate à Fome. O que é [acessado 2007 abr 12]. Disponível em: http://www.mds.gov.br/bolsafamilia/ o_programa_bolsa_familia/o-que-e

7. Santana JA. A evolução dos programas de transferência de renda e o Programa Bolsa Família [acessado 2008 set 11]. Disponível em: http://www. abep.nepo.unicamp.br/SeminarioPopulacaoPobreza Desigualdade2007/docs/SemPopPob07_1019.pdf 
8. Brasil. Ministério do Desenvolvimento Social e Combate à Fome. Ministério da Educação. Ministério da Saúde. As condicionalidades no contexto do Programa Bolsa Família. [acessado 2008 nov 25]. Disponível em: http://www.congemas.org.br/condicionalidades1.pdf

9. Morris SS, Olinto P, Flores R, Nilson EAF, Figueiró AC. Conditional cash transfers are associated with a small reduction in the rate of weight gain of preschool children in northeast Brazil. J Nutr 2004; 134:2336-2341.

10. Brasil. Ministério da Saúde. Secretaria de Atenção à Saúde. Departamento de Atenção Básica. Avaliação do Programa Bolsa-Alimentação. Primeira Fase. Série C. Projetos, Programas e Relatórios. Brasília, DF; 2004.

11. Comissão Nacional Sobre Determinantes Sociais da Saúde. As causas sociais das iniquidades em saúde no Brasil. Relatório final da Comissão Nacional Sobre Determinantes Sociais da Saúde (CNDSS). Abril, 2008.

12. Instituto Brasileiro de Geografia e Estatística (IBGE). Dados Populacionais. [acessado 2007 mar 8]. Disponível em: www.ibge.gov.br/cidadesat/default.php

13. Programa das Nações Unidas para o Desenvolvimento (PNUD). Atlas do Desenvolvimento Humano. 2003. [acessado 2007 jun 20]. Disponível em: http://www.pnud.org.br/atlas/

14. Biesalski HK, Erhardt JG. Diagnosis of nutritional anemia. Laboratory assessment of iron status. In: Kraemer K, Zimmermann MB. Nutritional Anemia. Switzerland: Sight and Life Press; 2007. p. 37-43.

15. Gleason G, Scrimshaw N. An overview of the functional significance of iron deficiency. In: Kraemer K, Zimmermann MB. Nutritional Anemia. Switzerland: Sight and Life Press; 2007. p. 45-58.

16. Jelliffe DB. The assessment of the nutritional status of the community. World Health Organization, Monograph Series, $n^{\circ}$ 53, Geneva, 1966.

17. World Health Organization (WHO). WHO Multicentre Growth Reference Study Group. WHO Child Growth Standards: length/height-for-age, weightfor-age, weight-for-length, weight-for-height and body mass index-for-age: methods and development. Geneva: World Health Organization, 2006. [acessado 2006 mai 25]. Disponível em: www.who. int/childgrowth/standards/em/index.html

18. World Health Organization (WHO). Physical Status: The Use and Interpretation of Anthropometry - Report of a WHO Expert Committee. Geneva, 1995. [acessado 2008 ago 5]. Disponível em: http:// whqlibdoc.who.int/trs/WHO_TRS_854.pdf
19. Conselho Nacional de Saúde. Resolução no 196 de 10 de outubro de 1996. [acessado 2009 mar 2]. Disponível em: http://conselho.saude.gov.br/docs/ Resolucoes/Reso196.doc

20. Fonseca AMM, Roquete C. Proteção social e programas de transferência de renda: o bolsa-família. In: Viana ALD, Elias PEM, Ibañez N. Proteção social dilemas e desafios. São Paulo: Editora Hucitec; 2005. p. 123-149.

21. Brasil. Ministério da Saúde. DATASUS. Estado nutricional dos beneficiários do Programa Bolsa Família. [acessado 2009 jan 12]. Disponível em: http:/ /bolsafamilia.datasus.gov.br/w $3 \mathrm{c} / \mathrm{tab}$.asp?Tp Acao $=0$

22. Vitolo MR, Gama CM, Bortolini GA, Campagnolo PDB, Drachler ML. Alguns fatores associados a excesso de peso, baixa estatura e déficit de peso em menores de 5 anos. J Pediatr 2008; 84(3):251-257.

23. Zöllner CC, Fisberg RM. Estado nutricional e sua relação com fatores biológicos, sociais e demográficos de crianças assistidas em creches da Prefeitura do Município de São Paulo. Rev Bras Saúde Matern Infan 2006; 6(3):319-328.

24. Magalhães KA. Programa Bolsa Família: operacionalização, integração e desafios à emancipação de famílias em situação de vulnerabilidade social. [dissertação]. Viçosa (MG): Universidade Federal de Viçosa; 2008.

25. Instituto Brasileiro de Análises Sociais e Econômicas (IBASE). Repercussões do Programa Bolsa Família na segurança alimentar e nutricional das famílias beneficiadas. Relatório técnico (preliminar). Rio de Janeiro, 2008. [acessado 2008 out 30]. Disponível em: http://www.ibase.br/userimages/ibase _bf_sintese_site.pdf

Artigo apresentado em 14/09/2009

Aprovado em 06/01/2010

Versão final apresentada em 27/01/2010 